\title{
Are mother-child similarities in value orientations related to mothers' parenting? A comparative study of American and Romanian mothers and their adolescent children
}

\author{
Mihaela Friedlmeier ${ }^{1}$ and Gisela Trommsdorff ${ }^{2}$ \\ 'Psychology Department, Grand Valley State University, Allendale, MI, USA \\ ${ }^{2}$ Psychology Department, University of Konstanz, Konstanz, Germany
}

\begin{abstract}
The current study investigated similarities between mothers' and their adolescent children's individualistic and collectivistic values by analysing data from 337 American mother-adolescent dyads and 100 Romanian mother-adolescent dyads. The two countries differ in the degree of socioeconomic and political changes as well as quality of intergenerational relationships, therefore differences in the extent of shared values between mothers and adolescents were expected. Similarities in value orientations between mothers and adolescents may be a result of actual transmission processes, but it may also be due to influences from the general value context in their society, an aspect often called Zeitgeist influence or cultural stereotype. Adolescents' perception of maternal parenting behaviours was hypothesized to moderate the similarities between mothers and adolescents. Hierarchical regression analyses revealed that collectivistic values were transmitted more than individualistic values and that stronger value similarities were found within biological dyads compared to randomly assigned dyads or within the peer group. Additionally, maternal acceptance and control moderated the similarities between mothers and adolescents, and associations between perceived maternal parenting and adolescents' values were revealed.
\end{abstract}

Kcywords: Intergenerational similarities; Values; Parenting; Culture.

Adolescence is the formative phase for establishing values and cultural orientations (Erikson, 1968; Rohan \& Zanna, 1996). The importance

Correspondence should be addressed to Mihaela Friedlmeier, Grand Valley State University, Psychology Department, Allendale, MI, 49401-9403, USA.

E-mail: friedlmm@gvsu.edu 
adolescents give to certain values may be explained by transmission processes, which are part of one's socialization (e.g., Grusec \& Goodnow, 1994), with parents seen as primary socialization agents or "the front-line troops" (Smith, 1983, p. 13). Studying adolescents' values is also important because they often act as a mediator between cognitions and behaviours (McGillicuddy-De Lisi \& Sigel, 1995), suggesting the central role of personal values in adolescents' positive development. At the same time, it is widely accepted now that children play an active role in their own socialization, and this active contribution may be especially strong during adolescence compared to earlier stages of development. Thus far, most studies in psychology that have looked at intergenerational value transmission have focused on Western societies. This implies certain shortcomings since value transmission takes place in a certain sociocultural context, which can be more or less stable, with different salience given to the contents of the values to be transmitted (Trommsdorff, 2009). It was the first aim of this study to examine the extent of value transmission in mother-adolescent dyads by comparing two countries with a different experience of social change, the United States and Romania.

Although some studies have found (modest) support for the influence of gender constellation on value transmissions between parents and children (e.g., Boehnke, Ittel, \& Baier, 2002; Roest, Dubas, \& Gerris, 2010), the current study focused only on mothers and their adolescent children. However, not all mother-adolescent similarities in values are a result of truly family influences; they may reflect a cultural stereotype, meaning the shared cultural and normative influence leading to typical responses of individuals (Cronbach, 1955). It was the second aim of this study to investigate the influence of a cultural stereotype, an aspect that has received little attention in the literature.

Despite the considerable interest in the process of value transmission, little is actually known about the conditions, often labelled transmission belts (Schönpflug, 2001), that may influence the similarity between mothers and adolescents. While some studies have investigated the influence of parenting style as reported by parents, less emphasis has been placed on how adolescents view maternal parenting (Kakihara \& Tilton-Weaver, 2009). The recipients of value transmission are adolescents, who are still in the process of value formation and acquisition; therefore, moderators may play an even stronger role compared to later stages when individuals already have a value system in place and personal experiences have shaped their values. Thus, the third aim of this study was to investigate whether adolescents' perception of maternal parenting is related to the extent of similarities between their own values and those of their mothers. Examining mother-adolescent relationships in the United States and Romania from the perspective of value similarities offers an opportunity to 
better understand intergenerational relationships in two different cultural environments.

\section{Context and content of intergenerational value similarities}

The first aim of this study was to investigate similarities in individualistic and collectivistic values between mothers and adolescent children in the USA and Romania. Individualism captures the values of self-direction, stimulation, and hedonism whereas collectivism consists of values of security, conformity, and tradition (Schwartz \& Bilsky, 1990). The impact of social, demographic and economic changes on intergenerational relations can also include changes in the similarities and differences between parents and children with respect to values (e.g., King \& Elder, 1997; Trommsdorff, 2006). While the United States is a more stable society, Romania is dealing with tremendous social, political and economic changes after the breakdown of communism. During the communist rule in Eastern Europe, fostering collectivism was a critical childrearing goal in childcare centres and schools, which was understood as cultivating a concern for the group, encouraging unquestioning obedience to authority, and developing subordination of the self in favour of group interests and demands (Ispa, 2002). Nowadays, one of the most salient characteristics in these countries, including Romania, is the shift from collectivist forms of social organizations and mentality toward individualist forms (see Reykowski, 1994, for a discussion). Acculturation studies on intergenerational value similarities suggest that in a discontinuous cultural context, parents are presumably less motivated to transmit their own standards and values to the next generation under the assumption that these will not be adaptive for their children in the future (Schönpflug, 2001). Although no data are available with regard to mothers' motivation to transmit their own values, by comparing American and Romanian motheradolescent dyads this study aimed to shed more light on the issue of intergenerational similarities during times of drastic social change. Romanian mothers who participated in this study have grown up in an autocratic social system (about $65 \%$ of them were in their early 30 s when communism collapsed) while their children were socialized under conditions influenced by the transition from a centrally planned to a market-type economy. One may argue here that Romanian adolescents may form their values according to their experience of the new societal structures, thus leading to less mother-adolescent value similarities in the Romanian dyads compared to the American dyads.

Additionally, several researchers investigated the issue of selective transmission, starting with Troll and Bengtson (1979), who showed that parents and children may be especially similar on religious and political values, and later with Kohn (1983), who showed a lack of similarity in core 
values such as responsibility, good manners and self-control. In a Dutch sample, Roest et al. (2010) found no parent to child transmission of hedonistic values (seen as part of individualistic values) whereas both parents transmitted their values of work as duty to their adolescent children. Drawing from a social evolutionary perspective, Schönpflug (2001) revealed more transmission of collectivistic values than individualistic, these being values that serve the in-group and enable co-operation. The salience hypothesis suggests that parents transmit those values to their children that are salient to them (Pinquart \& Silbereisen, 2004). However, although more recent studies have addressed the issue of selectivity or salience (e.g., Roest et al., 2010), we are not aware of any study that examined this from a cultural perspective.

According to gender role models of socialization theory, fathers transmit their values mostly to their sons and mothers mostly to their daughters (Vollebergh, Iedema, \& Raaijmakers, 2001), expecting stronger parent-tochild value transmission to take place within same-sex dyads. However, mixed results are found in the literature, with some studies bringing evidence for the same-sex parent-child transmission (Boehnke et al., 2002) while others found no gender effect (Whitbeck \& Gecas, 1988). Although only mothers participated in the current study, we were able to test whether more similarities existed in the mother-daughter dyads compared to mother-son dyads. However, testing gender effects was not a main focus of this paper.

The second aim of this study was to examine whether the similarities between mothers' and adolescents' values were a result of truly maternal influences or whether they reflected a cultural stereotype. Mothers are important socialization agents and empirical evidence has demonstrated their impact on children's value orientations; however, not all parent-child similarities can be regarded as an evidence of parental influence (Seginer \& Vermulst, 2001). The similarity within the family might be influenced (or biased) by a cultural stereotype or Zeitgeist, a term first used by Cronbach (1955) that refers to a shared cultural or normative influence leading to typical responses of individuals. Only a few studies have investigated this idea (e.g., Boehnke, 2001; Knafo \& Schwartz, 2003). For instance, Boehnke (2001) found value similarities between parents and adolescents as well as between non-kin adult-adolescent dyads. In the current study, we expected that family influences (mothers' values, perceived parenting) would have stronger impact on adolescents' value orientations compared to random child and non-kin adults.

\section{Parenting as moderator of intergenerational value similarities}

Much of the research on parenting has focused on what parents report they do and how this affects adolescents' adjustment (Kakihara \& Tilton-Weaver, 
2009). Still, little is known about how adolescents view parental behaviours and how this perception affects their value orientations. In the current study we focused on adolescents' perception of maternal control and acceptance. As measured in the current study, maternal acceptance comprises warmth, support and estimation. A successful value transmission is realized when parents are supportive and affectionate toward their children (Smith, 1983). Warmth in the parent-child relationship may make children eager to be similar to or please their agent of socialization (Grusec, Goodnow, \& Kuczynski, 2000).

More studies published in the last decade have distinguished between psychological and several aspects of behavioural control, a distinction which has proved helpful in disentangling some of the complexity surrounding the concept of parental control and its associations with child development (e.g., Barber, 1996; Barber, Olsen, \& Shagle, 1994). According to Barber (1996), psychological control refers to attempts to control behaviours by manipulating adolescents' emotions, thoughts or ideas whereas behavioural control creates a regulating structure through supervision, setting limits, and enforcing household rules. Grolnick and Pomerantz (2009) disagreed with this multiple-forms approach to parental control, and proposed two dimensions of parenting: (1) control, which comprises pressure, intrusion and domination of children's feelings, thoughts and behaviour; and (2) structure (guidance) of behaviours, feelings and thoughts. Interestingly, Kakihara and Tilton-Weaver (2009) showed that at high levels, adolescents did not differentiate between psychological and behavioural control, both being seen as intrusive especially when control was applied to the domains that adolescents considered under personal control.

Distinguishing between control and structure helps identify the mechanisms by which parenting shapes children (Grolnick \& Pomerantz, 2009). According to the self-determination theory (Ryan \& Deci, 2000), children have a need for autonomy, relatedness and competence, which are critical for their healthy development, and parental structure facilitates the development of these three components by providing feedback (Grolnick \& Pomerantz, 2009). Not providing this support and creating a chaotic environment may undermine children's competence (Skinner, Johnson, \& Snyder, 2005). Parental control restricting these needs could undermine adolescents' emotional and social development. Reactance theory (Brehm, 1966) argues that people experience psychological reactions when free choice over their own behaviour is threatened.

These are new developments in the approach on parenting, and not much consideration has yet been given to the cultural context, which may moderate the link between perceived parenting and adolescents' outcomes (Kakihara \& Tilton-Weaver, 2009). For instance, Japanese and Korean 
adolescents, but not German and American adolescents, felt neglected by their parents when they experienced low control (Rohner \& Pettengill, 1985; Trommsdorff, 1985). In cultural contexts in which independence and autonomy are highly valued, parental control may be less approved by adolescents than in cultures in which interdependence is highly valued (Rudy \& Grusec, 2001). Independence is typical for parent-child relations in the United States; therefore, high level of control here may undermine the similarity between adolescents' and mothers' values. Childrearing practices in Eastern Europe have been changing gradually over the years since the breakdown of communism (Robila, 2004). However, teaching obedience is still very important (at least from the perspective of the parents), the child being expected to listen to the parents and there is little room to negotiate with the parents (Robila, 2004). Therefore, based on the existing literature we argue that higher level of control may enhance the transmission.

To summarize, the present study examined similarities and differences between Romanian and American mothers and adolescents regarding individualistic and collectivistic values. Due to abrupt changes in Romania, we expected fewer similarities in the Romanian mother-adolescent dyads compared to the American dyads (hypothesis 1). The impact of the cultural stereotype on the mother-child value similarities was also examined, and we expected that family influences would be stronger than the influence of a cultural stereotype (hypothesis 2). We also expected that in both countries the similarities between mothers' and adolescents' values would be stronger when adolescents perceived their mothers as acceptant (hypothesis 3a). Furthermore, we hypothesized that high perceived maternal control would undermine the similarities in values between American mothers and children, but might enhance similarities in the Romanian dyads (hypothesis $3 b$ ).

\section{METHOD}

\section{Sample}

The sample included 437 mother-adolescents dyads (337 dyads in the USA and 100 in Romania). Participants in the USA were all European Americans who resided in a middle-large Midwestern city and its surrounding municipalities. Romanian participants were recruited from a middle-size town in the north-eastern region.

The mean age of the adolescents in the USA was 16.32 years $(S D=1.41$, ranging from 13 to 18 years) and 15.5 years old in Romania $(S D=1.1$, ranging from 14 to 17 years). American adolescents were significantly older than Romanian adolescents, $F(1,433)=34.40, p<.001$. The main effect of gender and the interaction effect between gender and culture were not 
significant. The adolescent sample in the USA was comprised of $35.9 \%$ $(n=121)$ males and $64.1 \%$ females $(n=216)$. In Romania, 52 girls and 48 boys participated in the study. The two cultural samples differed in the distribution of gender, $\chi^{2}(1)=4.75, p<.05$. Due to these differences between the two samples, adolescents' age and gender were controlled for in the data analyses. All children in both countries were living in the same household with their parents.

American mothers were significantly older $(M=45.17, S D=5.60)$ than Romanian mothers $(M=42.8$ years, $S D=4.4), t(435)=3.77, p<.001$. Of the Romanian mothers, $24 \%$ finished vocational or complementary school, $60 \%$ graduated from high school, and $16 \%$ had a college degree. In the American sample, $49 \%$ of the mothers had a high-school education, $15.5 \%$ completed a 2 -year vocational school, and $35.5 \%$ had college education or higher. The two cultural samples of mothers differed in the distribution of education, $\chi^{2}(3)=65.56, p<.001$. With regard to employment status, $65.2 \%$ of the mothers in the American sample were employed full time and $34.8 \%$ part time, while $89 \%$ of the Romanian mothers were employed full time, none of them having a part-time job. The distribution of employment status differed in the two cultural samples, $\chi^{2}(1)=42.51, p<.001$. We looked at the correlations between adolescents' value orientations and those of the mothers separated by education level and found no significant differences.

\section{Procedure}

We first contacted school principals and high-school teachers asking them to assist us in recruiting families with adolescents. Those who responded helped us send information packets containing the purpose of the study and a brief form with contact information to mothers. Mothers who agreed to participate (response rate was generally lower than $40 \%$ in the USA and around $75 \%$ in Romania) sent the signed form back to school through their adolescent child. In the USA, some families were also contacted through church groups and YMCA sport groups. In both countries, a structured face-to-face interview with the mother was carried out in the respondent's home after an appointment was scheduled over the phone. Mothers signed a consent form and a parental permission form for their participating child before the interview. Adolescents signed an assent form. During the home visit, adolescents in the USA answered the questionnaires independently in another room while their mothers were interviewed. In Romania, adolescents filled out the questionnaire in the classrooms under the supervision of the research assistant. Adolescents took around 40-45 minutes to complete their questionnaires. The interviews with the mothers took an average of 75 minutes. Each participant was given a debriefing letter 
and a small gift at the end of the interview. The data were collected between 2005 and 2007 in the USA and in 2001 in Romania.

\section{Measures}

The instruments comprised a large number of self-report questions, which were assessed in both samples. The questions were translated from English to Romanian by a graduate student majoring in English and an independent back-translation from Romanian to English was carried out by another student. The investigator compared the two versions of translation and-if necessary-modifications were made after consulting with the translator.

Individualism-collectivism. A short version of Schwartz and Bilsky's (1990) instrument was used, which consisted of 13 items. It is a well-known instrument used to assess values in different cultures. In the current study a short version was utilized that was developed as part of the COLINDEX (Chan, 1994). Subjects were asked to judge each value regarding the extent to which it constituted a guiding principle in their lives. A 5-point scale ranging from 1 (not important at all) to 5 (very important) was applied. Seven items assessed individualism and included items related to self-direction, stimulation, and hedonism, e.g., independence (self-reliance, choosing your own goals and interests), an exciting life (stimulating experiences). Six items assessing collectivist values included items related to security, conformity, and tradition, e.g., honouring your parents and elders (showing respect), politeness (courtesy, good manners). Internal consistencies were good in both countries: in the USA, individualism: $\alpha$ (adolescents) $=.74$ and $\alpha$ (mothers) $=.76$; collectivism: $\alpha$ (adolescents) $=.76$ and $\alpha$ (mothers) $=.71$. For the Romanian sample, individualism: $\alpha$ (adolescents) $=.74$ and $\alpha$ (mothers) $=.77$; collectivism: $\alpha$ (adolescents) $=.75$ and $\alpha$ (mothers) $=.64$.

Adolescents' perception of mothers' parenting style. The Parental Acceptance-Rejection Questionnaire (PARQ; Rohner, Rohner, \& Roll, 1980) as well as the Control-Supplement were used. Only two scales were selected for this study: acceptance (10 items, e.g., my mother treats me gently and with kindness) and control (6 items, e.g., my mother is always telling me how I should behave). Items were rated on a 4-point Likert-type scale ranging from 1 (almost never true) to 4 (almost always true). Adolescent were asked about their mothers' parenting behaviours. One item was dropped from the acceptance scale for both samples because of its low item-scale correlation (My mother is interested in the things I do). Acceptance scale reached an $\alpha=.89$ in the US sample, and $\alpha=.85$ in the Romanian sample. For the control scale, alphas were .74 in both samples. 


\section{Method of analysis}

Multiple regression analyses were employed in order to examine the predictive relationship between mothers' variables and children's variables. The main emphasis in these analyses was to show the extent to which mothers' and adolescents' values were similar using as predictors mothers' variables (value orientations) alone and in combination (interaction) with moderator variables, such as parenting style. According to Baron and Kenny (1986), in order to analyse the moderator effect, the interaction term between the values of mother and the moderator variable (e.g., acceptance) was introduced in the regression analyses to predict the values of the adolescents. The continuous predictor variables were each centred on their mean before the interaction variable was computed (Aiken \& West, 1991). In order to examine whether associations between variables worked differently in the two cultures, two- and three-way interaction terms between cultures and the predictor variables were created (culture was coded as a dummy variable).

In order to test the cultural stereotype, we followed the strategy proposed by Boehnke, Hadjar, and Baier (2009) by creating pseudo motheradolescent dyads as well as adolescent-peer dyads. Each adolescent was assigned the score of a mock peer and a mock mother. When adolescents' values correlate positively with values of randomly selected peers and mothers, this is not a sign of family influences, but an influence of a cultural stereotype. These pseudo variables (as well as their interaction with culture) were entered as control variable into the regression models in order to separate their effect from those of the intrafamilial value influence (see Boehnke et al., 2009, p. 447).

To summarize, we calculated the amount of explained variance in the adolescents' value orientations by entering the random mother, random child and the interactions with culture (Block 1), mother's value orientation and parenting variables (Block 2), two-way interactions between value and parenting, value and culture, parenting and culture (Block 3), and three-way interaction between value, parenting and culture (Block 4). The three-way interactions between culture, value orientations and parenting never reached significance, thus they are not documented in the table. Analyses were performed separately per value orientation (individualistic/collectivistic). All analyses were controlled for the age and gender of adolescents.

\section{RESULTS}

\section{Descriptive analyses for study variables}

Mothers in both countries scored significantly higher on collectivism as compared to the adolescents, $F(1,873)=40.15, p<.001$, whereas adoles- 
cents scored significantly higher on individualism, $F(1,873)=94.30$, $p<.001$ (see Table 1). No significant cultural differences were found for values and adolescents' perception of maternal parenting.

\section{Intergenerational similarities in value orientations}

Mothers' individualism entered in Step 2 significantly increased the portion of explained variance in adolescents' individualism $(\beta=0.12, p<.05)$ and culture moderated the relationship between mothers' individualism and adolescents' individualism (see Table 2). Further analyses showed that only in the USA are mothers and adolescents more similar in their individualistic values $(\beta=0.18, p<.01)$, but not in Romania $(\beta=0.001$, $n$ s; see also Figure 1). Further analyses separated by gender showed transmission of individualism in the mother-son dyads in both countries and in the motherdaughter dyads in the USA, but not in Romania.

With regard to the second value orientation, collectivism of mothers was significantly and positively related to collectivism of adolescents $(\beta=0.23$, $p<.001$; see Table 2) and the relationship was not moderated by culture. Further analyses by gender showed stronger intergenerational similarities in the mother-son dyads $(\beta=0.29, p<.001)$ compared to mother-daughter dyads $(\beta=0.20, p<.001)$. Overall, stronger intergenerational similarities were found for collectivistic values compared to individualistic values $(z=1.98, p=.05)$. In conclusion, hypothesis 1 was only partially confirmed since intergenerational similarities were stronger for individualistic values in the American dyads, but they were similar for collectivistic values.

The cultural stereotype variables (entered in Step 1) explained only 1 to $2 \%$ of the variance in the adolescents' value orientations, and none of the models were statistically significant from 0 (see Table 2). Thus, hypothesis 2 was confirmed in that variance explained by family influence (Step 2) was stronger.

\section{Parenting as moderator}

Maternal acceptance did not moderate the relationship between mothers' and children's individualistic and collectivistic values (see Table 2); therefore, hypothesis $3 \mathrm{a}$ was rejected. Perceived maternal control moderated the relationship between mothers' collectivism and adolescents' collectivism $(\beta=-0.10, p<.05)$. Following Cohen and Cohen (1983), we used three values of the moderator variable (control), corresponding to the centred mean, one standard deviation above, and one standard deviation below the mean. These values were substituted in the regression equations to generate a series of simple regression equations of the dependent variable on the predictor variable. These analyses showed that the similarity between 
TABLE 1

Mean values and intercorrelations for mothers' and adolescents' value orientations and parenting

\begin{tabular}{|c|c|c|c|c|c|c|c|c|}
\hline & $\begin{array}{c}U S A \\
(n=337) \\
M(S D)\end{array}$ & $\begin{array}{c}R O \\
(n=100) \\
M(S D)\end{array}$ & 1 & 2 & 3 & 4 & 5 & 6 \\
\hline 1. Indivichualism $A$ & $4.13(0.53)$ & $4.13(0.55)$ & - & $.16^{\dagger}$ & .00 & -.06 & .09 & -.13 \\
\hline 2. Collectivism $A$ & $4.06(0.60)$ & $4.16(0.57)$ & $.36^{* * *}$ & - & -.04 & $.18^{\dagger}$ & $.32^{* *}$ & -.10 \\
\hline 3. Individualism $M$ & $3.74(0.54)$ & $3.64(0.67)$ & $.16^{* *}$ & $.12^{*}$ & - & $.25^{*}$ & .03 & -.07 \\
\hline 4. Collectivism $M$ & $4.36(0.47)$ & $4.40(0.43)$ & .05 & $.27^{* * *}$ & $.38^{* * *}$ & - & .03 & .07 \\
\hline 5. Acceptance & $3.47(0.53)$ & $3.49(0.47)$ & .07 & $.28^{* * *}$ & .02 & .04 & - & $-.27^{* *}$ \\
\hline 6. Control & $2.86(0.60)$ & $2.76(0.69)$ & $.10^{\dagger}$ & .02 & -.07 & $.21^{* * *}$ & $-.21^{* * *}$ & - \\
\hline
\end{tabular}

Notes: $\mathrm{A}=$ Adolescents; $\mathrm{M}=$ Mothers. The intercorrelations for Romanian mother-adolescent dyads are presented above the diagonal and for American dyads below the diagonal. ${ }^{\dagger} p<.10 ;{ }^{*} p<.01 ;{ }^{* *} p<.05 ;{ }^{* * *} p<.001$. 


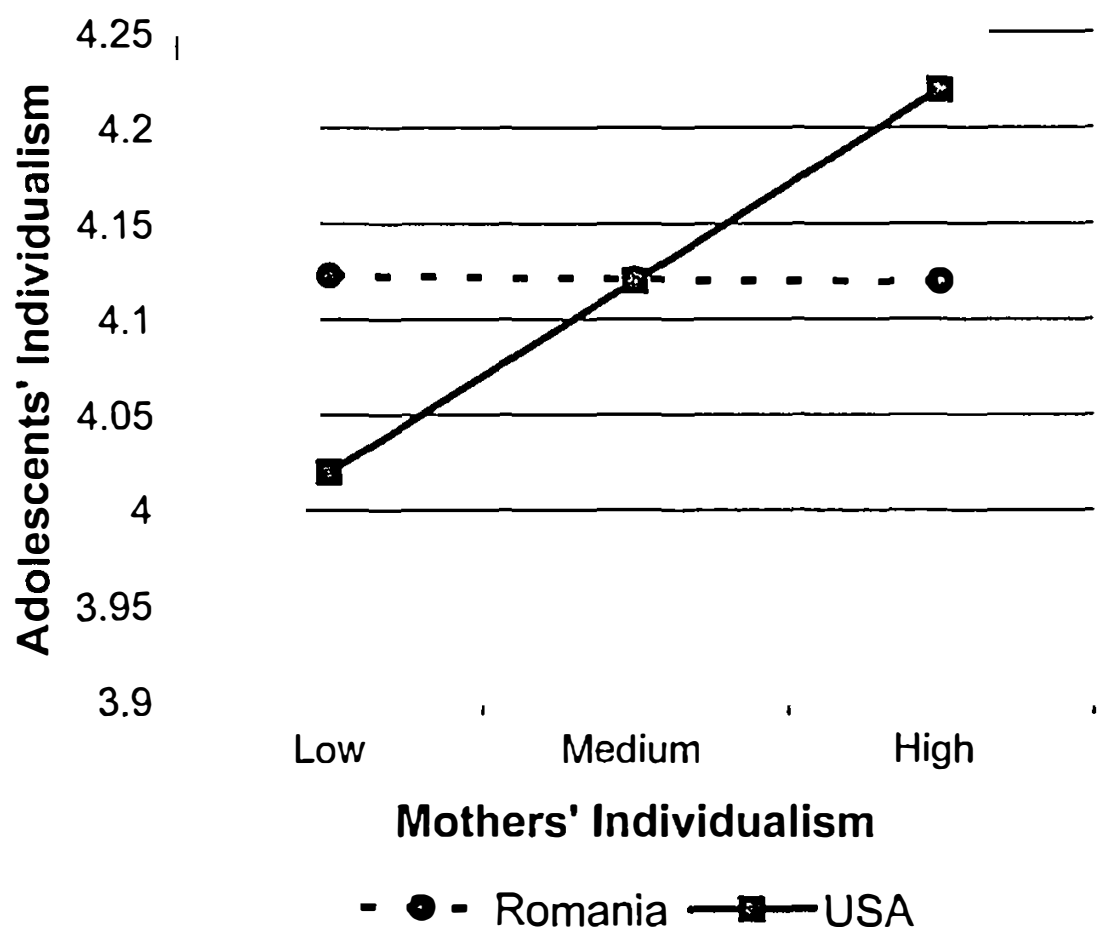

Figure 1. Intergenerational similarities in individualistic values in Romania and the USA.

\section{TABLE 2}

Hierarchical regression for mothers' values and perceived parenting predicting adolescents' values

$$
\text { Individualism }
$$

$$
\beta \quad \Delta R^{2} \quad \Delta F
$$

Collectivism

\begin{tabular}{|c|c|c|c|c|c|c|}
\hline Step 1 & & .02 & 1.08 & & .03 & 1.01 \\
\hline Culture & 0.00 & & & 0.07 & & \\
\hline Value random mother (VRM) & $-0.10^{\dagger}$ & & & -0.01 & & \\
\hline Value random child (VRC) & -0.05 & & & 0.07 & & \\
\hline Culture $\times$ VRM & 0.01 & & & -0.04 & & \\
\hline Culture $\times$ VRC & 0.05 & & & -0.06 & & \\
\hline Step 2 & & .02 & $3.11^{*}$ & & .14 & $23.47 * * *$ \\
\hline Value mother & $0.12^{*}$ & & & $0.23^{* * *}$ & & \\
\hline Acceptance & 0.08 & & & $0.29 * * *$ & & \\
\hline Control & 0.07 & & & -0.02 & & \\
\hline Step 3 & & .03 & $2.08^{\dagger}$ & & .01 & 1.22 \\
\hline $\mathrm{C} \times$ Value mother & $-0.11^{*}$ & & & -0.03 & & \\
\hline Value $\mathbf{M} \times$ Acceptance & 0.04 & & & -0.02 & & \\
\hline Value $\mathrm{M} \times$ Control & -0.05 & & & $-0.10^{*}$ & & \\
\hline $\mathrm{C} \times$ Acceptance & -0.02 & & & 0.03 & & \\
\hline $\mathrm{C} \times$ Control & $-0.11^{*}$ & & & -0.03 & & \\
\hline Total $R^{2}$ & & .07 & & & .18 & \\
\hline
\end{tabular}

$$
\beta \quad \Delta R^{2} \quad \Delta F
$$

Notes: Analyses were controlled for adolescents' age and gender. Culture was entered as dummy variable (USA $=0$; Romania $=1$ ). ${ }^{\dagger} p<.10 ;{ }^{*} p<.05 ;{ }^{* * *} p<.001$. 
collectivism of the mother and that of the adolescent is greatest in the group of low-controlling mothers $(\beta=0.49, p<.001)$, followed by mediumcontrolling mothers $(\beta=0.36, p<.01)$, and lastly, high-controlling mothers $(\beta=0.23, p<.01$; see Figure 2$)$. However, these results support hypothesis $3 \mathrm{~b}$ only partially since no culture-specific results were found. Additional gender analyses showed two moderating effects for girls, but not for boys. Perceived maternal acceptance moderated the similarities in collectivism between mothers and daughters $(\beta=0.13, p<.05)$, with stronger similarities in the group of high-acceptance mothers. Perceived control moderated the mother-daughters similarities in individualism $(\beta=-0.13, p<.05)$, with stronger similarities in the group of low-controlling mothers.

While no hypotheses were formulated, the findings showed some interesting direct relations between perceived maternal parenting and adolescents' values. Higher perceived maternal acceptance was associated with stronger importance attributed to collectivist values by adolescents $(\beta=0.29, p<.001$; see Table 2$)$. Perceived maternal control showed opposite effects on adolescents' individualistic values (see Table 2 and Figure 3): In the USA, a significant positive relationship was found $(\beta=0.11, p<.05)$ whereas in Romania a negative relationship, but not significant, was revealed $(\beta=-0.11, p=.22)$. In other words, adolescents in the USA who perceived their mothers as controlling had stronger individualistic values.

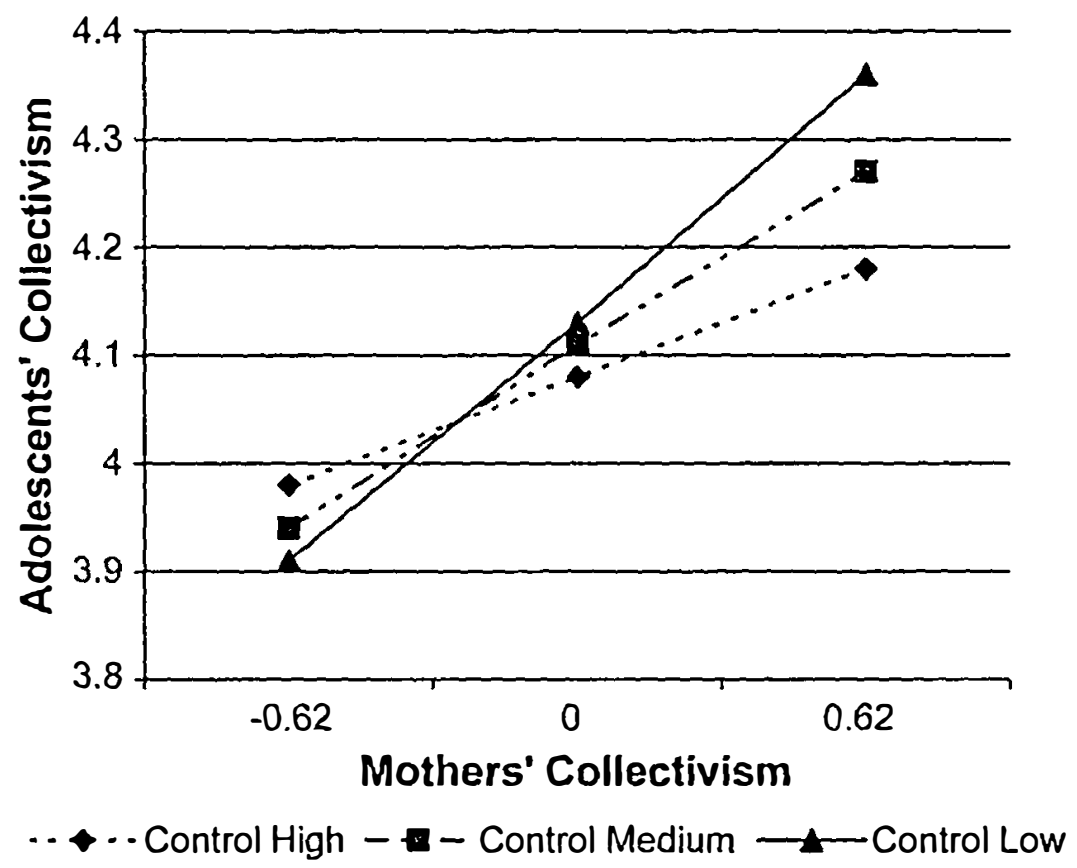

Figure 2. Intergenerational similarities in collectivistic values moderated by perceived maternal control. 


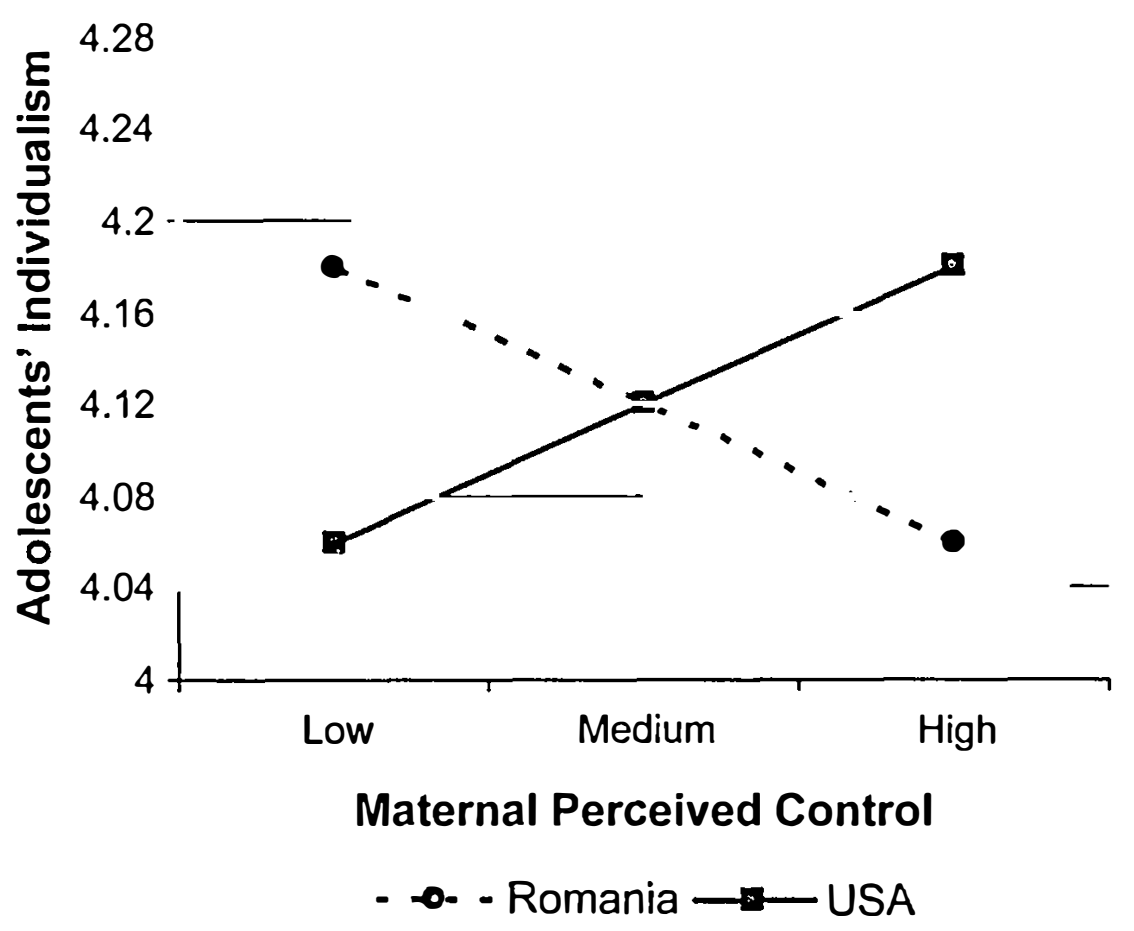

Figure 3. Predicting adolescents' individualistic values in Romania and the USA. Moderated by perceived maternal control.

\section{Discussion}

The current study investigated similarities in individualistic and collectivistic values between mothers and adolescent children by taking cultural context (Romania and the USA) and perceived maternal parenting (acceptance and control) into account. Values are important during adolescence and, although adolescents' conformity to parents is on the decline, the family remains the primary context for the socialization of values (Smith, 1983). Due to the abrupt changes that took place in post-communist Romania, we expected fewer mother-adolescent similarities in value orientations in the Romanian sample compared to the American sample. While mothers and their adolescent children were similar in their collectivistic values in both countries, similarities in individualism were found only in the American sample; it should be mentioned that these similarities were not substantial. However, weak relations between mothers' and adolescents' values at this developmental stage do not necessarily mean a failed socialization; rather, long-term effects of transmission should be studied, especially since during adolescence a certain distance from the parents is considered appropriate for processes of identity formation (Ryan \& Deci, 2000). Overall, more similarities across cultures were found than differences with regard to value similarities. It may be the case that the developmental stage has more influence on which values are considered important, much more so than the degree of social change taking place in the society. 
Not all parent-child similarities are due to transmission processes, in part they are due to values prevailing in the social environment at a specific time point (e.g., Boehnke, 2001). Our analyses on the role of cultural stereotype showed that similarities within biologically related dyads are stronger than similarities within randomly assigned adultwoman-adolescent dyads or peer, thus pointing to a family contribution in the socialization of individualistic and collectivistic values. Although some studies revealed cultural stereotypes' effects on adolescents' values (such as family obligations values in Vedder, Berry, Sabatier, \& Sam, 2009), we failed to replicate these findings. Roest, Dubas, Gerris, and Engels (2009) revealed different outcomes for cultural stereotypes when using different measurement strategies (absolute difference scores, profile correlations or ordinary correlations). This is an aspect that needs closer examination in future research. Harris (1995) argued that, beyond infancy, parents make very little contribution to the socialization of their children, but peer groups play the dominant role. This is, however, not what our findings suggested.

In line with other studies (Knafo \& Schwartz, 2009; Roest et al., 2009; Schönpflug, 2001), we found stronger intergenerational similarities for collectivistic values than for individualistic values. This may be seen as evidence for the salience hypothesis (Pinquart \& Silbereisen, 2004), with more salient values being transmitted more. Although we did not specifically measure the salience of each value, on the basis of group mean level scores, mothers reported greater importance given to collectivistic values than to individualistic values. Knafo and Schwartz (2009) argued that adolescents accept more parental influence in the moral domain such as tradition and prosocial values than in the personal domain (e.g., self-direction, hedonism). These values from the moral, conventional domain may serve group maintenance; they have a more altruistic content and therefore can foster co-operation (Schönpflug, 2001). On the other hand, hedonism, stimulation, self-direction, and achievement values concern primarily personal issues (Schwartz, 1992) and they are less likely to be accepted by adolescents (Knafo \& Schwartz, 2009).

Certain parenting behaviours may enhance or hinder the similarities between mothers' and adolescents' values; while experiencing acceptance and support from the mother may lead to stronger similarities, adolescents who perceive their mothers as highly controlling may feel their autonomy threatened and look for more appropriate models (Grusec et al., 2000). Surprisingly, acceptance was not a relevant moderator of intergenerational similarities. This may be due to the fact that we only measured general parenting behaviour and did not specify the domains that are relevant for adolescents' development. In a recent study Grusec and Davidov (2010) proposed that socialization processes should be viewed from a domain 
perspective and that in each domain of social interaction children may accept different degrees of influence.

However, perceived maternal control moderated the relationship between collectivism of mothers and that of adolescents: the strongest similarities between mothers and adolescents were found when mothers were perceived as least controlling by their adolescent child, and the results were similar across the two cultural contexts. Higher levels of control can be interpreted as a threat to their autonomy, thus decreasing their motivation to comply with parental wishes (Grusec \& Goodnow, 1994). We did not find support for culture-specific impact of control on value transmission, thus possibly pointing to a universal interpretation of high level of control as intrusive (Kakihara \& Tilton-Weaver, 2009). More investigations are needed in order to clarify the meaning and functions of maternal control during adolescence in Eastern European countries.

Greater transmission in the mother-son dyad may be an indicator for the gender salience perspective. Presumably, collectivistic and individualistic values are more salient for sons. Furthermore, more moderating effects of maternal acceptance and control were found for girls compared to boys, suggesting that perhaps girls are more sensitive to aspects of their relationship with the mother.

Adolescents form their values partly based on their perception of parental behaviours (Roest, Dubas, \& Gerris, in press). Our findings also suggest that adolescents who perceive their mothers as acceptant and supportive emphasized values such as obedience, security and tradition. Furthermore, adolescents in the USA (not in Romania) who perceived their mothers as controlling also reported stronger individualistic values. Kasser, Ryan, Zax, and Sameroff (1995) showed that 18-year-olds with cold, controlling mothers were especially likely to endorse values of financial success, whereas teens with warm, democratic mothers were more focused on selfacceptance, affiliation, and community feelings. It is interesting, however, that maternal control has not been found to be related to individualism among Romanian adolescents. Further cross-cultural studies are needed in order to better understand these relationships.

One shortcoming of this study is the fact that the American sample consisted only of European Americans. Some studies showed differences between African-American and European-American adolescents in their perception and meaning of parental practices (Jackson-Newsom, Buchanan, $\&$ McDonald, 2008). Future studies should examine larger and more diverse samples in order to better understand the process of values transmission in different socioeconomic, cultural, and ethnic groups. Another limitation of this study is that it included only mothers. Friedlmeier (2006) found stronger similarities in collectivistic values between both parents and their sons compared to daughters in a Romanian sample. Future research should 
consider both maternal and paternal values, as well as agreement between parents (see Knafo \& Schwartz, 2003), when examining values transmission. In studies on value similarities, especially those involving countries going through abrupt social changes, it would be particularly interesting to see not only how parental characteristics affect the children, as was the case in the current study, but also how children influence their parents (Pinquart \& Silbereisen, 2004). With increasing age (and, consequently, an increase in power and status) children may have a stronger impact on their parents. From a developmental perspective, it is also important to examine developmental changes in adolescents' interpretations of parenting. Also, with increasing age, adolescents show decreasing conformity to parental influences raising the developmental question of optimal time span in adolescence for the transmission of values (Schönpflug, 2001). Further studies should examine intergenerational value transmission during early, middle and late adolescence. It is possible that the different method of administration of the adolescent measures (in their home for adolescents in the American sample and in school in Romania) introduced a certain bias due to the proximity of family members versus peer groups. Due to the cross-sectional design of the current study, no causal relations could be tested, a longitudinal study would be a better approach.

In spite of these limitations, the present study extends prior research in several ways. We examined similarities in value orientations between mothers and adolescent children in two different cultural contexts, while also looking at the influence of a cultural stereotype in the form of peer values and non-kin adult women. Overall, there is very little family research in Romania since most social science academic departments were closed during the 1970s and 1980s and currently this research is still at the beginning of its development (Robila, 2004). We found further support for stronger similarities between mothers and their adolescent children with regard to collectivistic values compared to individualistic values. Maternal influences are stronger than cultural stereotype influences. We also showed that perceived acceptance and control are relevant transmission belts for value orientations in both cultural contexts.

\section{REFERENCES}

Aiken, L. S., \& West, S. G. (1991). Multiple regression: Testing and interpreting interactions. Newbury Park, CA: Sage.

Barber, B. K. (1996). Parental psychological control: Revising a neglected construct. Child Development, 67, 3296-3319. 
Barber, B. K., Olsen, J. E., \& Shagle, S. C. (1994). Association between parental psychological control and behavioural control and youth internalized and externalized behaviors. Child Development, 65, 1120-1136.

Baron, R. M., \& Kenny, D. A. (1986). The moderator-mediator variable distinction in social psychological research: Conceptual, strategic, and statistical considerations. Journal of Personality' and Social Psychology, 51, 1173-1182.

Bochnke, K. (2001). Parent-offspring value transmission in a societal context. Journal of CrossCultural Psy'chology', 32, 241-255.

Boehnke, K., Hadjar, A., \& Baier, D. (2009). Value transmission and "zeitgeist" revisited. In U. Schönpflug (Ed.), Cultural transmission: Psychological, developmental, social, and methodological aspects (pp. 441-460). Cambridge, UK: Cambridge University Press.

Boehnke, K., Ittel, A., \& Baier, D. (2002). Value transmission and "Zeitgeist": An underresearched relationship. Sociale Wetenschappen, 45, 28-43.

Brehm, J. W. (1966). A theory of psychological reactance. New York, NY: Academic Press.

Chan, D. K.-S. (1994). COLINDEX: A refinement of three collectivism measures. In U. Kim, H. C. Triandis, C. Kagitcibasi, S.-C. Choi, \& G. Yoon (Eds.), Individualism and collectivism: Theory, method, and applications (pp. 200-210). Thousand Oaks, CA: Sage.

Cohen, J., \& Cohen, P. (1983). Applied multiple regression correlation analysis for the behavioral sciences (2nd ed.). Hillsdale, NJ: Lawrence Erlbaum Associates, Inc.

Cronbach, L. J. (1955). Processes affecting scores on "understanding of others" and "assumed similarity". Psychological Bulletin, 52, 177-193.

Erikson, E. (1968). Identity', youth and crisis. New York, NY: Norton.

Friedlmeier, M. (2006). Transmission of values within families in Romania. Doctoral dissertation, University of Konstanz, Konstanz, Germany. Retrieved from http://nbn-resolving.de/ urn:nbn:de:bsz:352-opus-55054

Grolnick, W. S., \& Pomerantz, E. M. (2009). Issues and challenges in studying parental control: Toward a new conceptualization. Child Development Perspectives, 3, 165-170.

Grusec, J. E., \& Davidov, M. (2010). Integrating different perspectives on socialization theory and research: A domain-specific approach. Child Development, 81, 687-709.

Grusec, J. E., \& Goodnow, J. J. (1994). Impact of parental discipline methods on the child's internalization of values: A reconceptualization of current points of view. Developmental Psy'chology, 30, 4-19.

Grusec, J. E., Goodnow, J. J. \& Kuczynski, L. (2000). New directions in analyses of parenting contributions to children's acquisition of values. Child Development, 71, 205-211.

Harris, J. R. (1995). Where is the child's environment? A group socialization theory of development. Psychological Review', 102, 458-489.

Ispa, J. M. (2002). Russian child care goals and values: From Perestroika to 2001. Early Childhood Research Quarterly, 17, 393-413.

Jackson-Newsom, J., Buchanan, C. M., \& McDonald, R. M. (2008). Parenting and perceived maternal warmth in European American and African American adolescents. Journal of Marriage and Family, 70, 62-75.

Kakihara, F., \& Tilton-Weaver, L. (2009). Adolescents' interpretations of parental control: Differentiated by domain and types of control. Child Development, 80, 1722-1738.

Kasser, T., Ryan, R. M., Zax, M., \& Sameroff, A. J. (1995). The relations of maternal and social environments to late adolescents' materialistic and prosocial values. Developmental Psychology, 31, 907-914.

King, V., \& Elder, G. H. J. (1997). The legacy of grandparenting: Childhood experiences with grandparents and current involvement with grandchildren. Journal of Marriage \& Family, $59,848-859$.

Knafo, A., \& Schwartz, S. H. (2003). Parenting and adolescents' accuracy in perceiving parental values. Child Development, 74, 595-611. 
Knafo, A., \& Schwartz, S. H. (2009). Accounting for parent-child value congruence: Theoretical considerations and empirical evidence. In U. Schönpflug (Ed.), Cultural transmission: Psychological, developmental, social, and methodological aspects (pp. 240-269). Cambridge, UK: Cambridge University Press.

Kohn, M. L. (1983). On the transmission of values in the family: A preliminary formulation. In A. C. Kerckhoff (Ed.), Research in sociology' of education and socialization (Vol. 4, pp. 3-12). Greenwich, CT: JAI.

McGillicuddy-De Lisi, A. V., \& Sigel, I. E. (1995). Parental beliefs. In M. H. Bornstein (Ed.), Handbook of parenting. Vol. 3: Status and social conditions of parenting (pp. 333-358). Mahwah, NJ: Lawrence Erlbaum Associates, Inc.

Pinquart, M., \& Silbereisen, R. K. (2004). Transmission of values from adolescents to their parents: The role of value content and authoritative parenting. Adolescence, 39, 82100.

Reykowski, J. (1994). Collectivism and individualism as dimensions of social change. In U. Kim, C. Kagitcibasi, S.-C. Choi, \& G. Yoon (Eds.), Individualism and collectivism: Theory, method, and applications (pp. 276-292). Thousand Oaks, CA: Sage.

Robila, M. (2004). Families in Eastern Europe: Context, trends and variations. Contemporary' Perspectives in Family Research, 5, 1-14.

Roest, A. M. C., Dubas, J. S., \& Gerris, J. R. M. (2010). Value transmissions between parents and children: Gender and developmental phase as transmission belts. Journal of Adolescence, 33, 21-31.

Roest, A. M. C., Dubas, J. S., \& Gerris, J. R. M. (in press). Children's value orientations as they traverse adolescence and emerge in adulthood: Alternative routes of parental transmissions. Journal of Research on Adolescence.

Roest, A. M. C., Dubas, J. S., Gerris, J. R. M., \& Engels, C. M. E. (2009). Value similarities among fathers, mothers, and adolescents and the role of a cultural stereotype: Different measurement strategies reconsidered. Journal of Research on Adolescence, 19, 812-833.

Rohan, M. J., \& Zanna, M. P. (1996). Value transmission in families. In C. Seligman, J. M. Olson, \& M. P. Zanna (Eds.), The psychology of values: The Ontario symposium on personality and social psychology' (Vol. 8, pp. 253-276). Mahwah, NJ: Lawrence Erlbaum Associates, Inc.

Rohner, E. C., Rohner, R. P., \& Roll, S. (1980). Perceived parental acceptance-rejection and children's reported behavioral dispositions. A comparative and intracultural study of American and Mexican children. Journal of Cross-Cultural Psychology, 11, 213-231.

Rohner, R. P., \& Pettengill, S. M. (1985). Perceived parental acceptance-rejection and parental control among Korean adolescents. Child Development, 56, 524-528.

Rudy, D., \& Grusec, J. E. (2001). Correlates of authoritarian parenting in individualist and collectivist cultures and implications for understanding the transmission of values. Journal of Cross-Cultural Psychology, 32, 202-212.

Ryan, R. M., \& Deci, E. L. (2000). Self-determination theory and the facilitation of intrinsic motivation, social development, and well-being. American Psychologist, 55, 68-78.

Schönpflug, U. (2001). Intergenerational transmission of values. The role of transmission belts. Journal of Cross-Cultural Psychology, 32, 174-185.

Schwartz, S. H. (1992). Universals in the content and structure of values: Theoretical advances and empirical tests in 20 countries. In M. P. Zanna (Ed.), Advances in experimental social psychology (pp. 5-65). New York, NY: Academic Press.

Schwartz, S. H., \& Bilsky, W. (1990). Toward a theory of the universal content and structure of values: Extensions and cross-cultural replications. Journal of Personality and Social Psychology, 58, 878-891.

Seginer, R., \& Vermulst, A. (2001). Family environment, educational aspirations, and academic achievement in two cultural settings. Journal of Cross-Cultural Psy'chology, 33, 540-558. 
Skinner, E., Johnson, S., \& Snyder, T. (2005). Six dimensions of parenting: A motivational model. Parenting: Science and Practice, 5, 175-235.

Smith, T. E. (1983). Parental influence: A review of the evidence of influence and a theoretical model of the parental influence process. In A. C. Kerckhoff (Ed.), Research in sociology of education and socialization (Vol. 4, pp. 13-45). Greenwich, CT: JAI Press.

Troll, L., \& Bengtson, V. (1979). Generations in the family. In W. R. Burr, R. Hill, F. I. Nye, \& I. L. Reiss (Eds.), Contemporary theories about the family: Research based theories (pp. 127161). New York, NY: Free Press.

Trommsdorff, G. (1985). Some comparative aspects of socialization in Japan and Germany. In I. R. Lagunes \& Y. H. Poortinga (Eds.), From a different perspective: Studies of behavior across cultures (pp. 231-240). Lisse, The Netherlands: Swets \& Zeitlinger.

Trommsdorff, G. (2006). Parent-child relations over the life span. A cross-cultural perspective. In K. H. Rubin \& O. B. Chung (Eds.), Parenting beliefs, behaviors, and parent-child relations. A cross-cultural perspective (pp. 143-183). New York, NY: Psychology Press.

Trommsdorff, G. (2009). Intergenerational relations and cultural transmission. In U. Schönpflug (Ed.), Cultural transmission: Psychological, developmental, social, and methodological aspects (pp. 126-161). Cambridge, UK: Cambridge University Press.

Vedder, P., Berry, J., Sabatier, C., \& Sam, D. (2009). The intergenerational transmission of values in national and immigrant families: The role of zeitgeist. Journal of Youth Adolescence, 38, 642-653.

Vollebergh, W. A. M., Iedema, J., \& Raaijmakers, Q. A. (2001). Intergenerational transmission and the formation of cultural orientations in adolescence and young adulthood. Journal of Marriage and the Family, 63, 1185-1198.

Whitbeck, L. B., \& Gecas, V. (1988). Value attributions and value transmission between parents and children. Journal of Marriage and the Family, 50, 829-840. 